\title{
CONCEPTS
}

\section{Medical Planning for Extended Remote Expeditions}

\author{
Kenneth V. Iserson, MD, MBA \\ From the Department of Emergency Medicine, The University of Arizona College of Medicine, Tucson, AZ.
}

\begin{abstract}
Remote extended expeditions often support scientific research and commercial resource exploration or extraction in hostile environments. Medical support for these expeditions is inherently complex and requires in-depth planning. To be successful, this planning must include substantial input from clinicians with experience in remote, emergency, and prehospital medicine and from personnel familiar with the proposed working environment. Using the guidelines discussed in this paper will help ensure that planners consider all necessary, medically relevant elements before launching an extended remote expedition.
\end{abstract}

The 10 key elements of a workable remote healthcare system are to:

1. Optimize workers' fitness

2. Anticipate treatable problems

3. Stock appropriate medications

4. Provide appropriate equipment

5. Provide adequate logistical support

6. Provide adequate medical communications

7. Know the environmental limitations on patient access and evacuation

8. Use qualified providers

9. Arrange for knowledgeable and timely consultations

10. Establish and distribute rational administrative rules

Planners using these guidelines may better be able to generate a strategy that optimizes the participants' health benefits, the expedition's productivity, and the expedition sponsor's cost savings.

\section{Introduction}

Remote travelers have long been concerned about their medical care. Alexander the Great traveled with his personal physician, Philippus, as he conquered the world. In the early twentieth century, Admiral Robert Peary took Dr Frederick Cook on his 1891 Greenland expedition, where he set Peary's fractured leg. Ernest Shackleton brought Eric Marshall on Antarctic expeditions as his chief surgeon/cartographer/surveyor. On his ill-fated 1912 expedition, Robert Falcon Scott chose Edward Wilson as his doctor/marine biologist/ornithologist.

Remote expedition medicine provides medical diagnosis and treatment to teams traveling to the developing

Corresponding author: Kenneth V. Iserson, MD, MBA, Department of Emergency Medicine, The University of Arizona College of Medicine, 4930 N. Calle Faja, Tucson, AZ 85718 (e-mail: kvi@u. arizona.edu). world or to remote geographical regions "where access to definitive medical care will involve prolonged evacuation over many hours or days." "The practice of remote medicine involves many challenges, including dealing with isolated environments, limited clinical diagnostic support and specialist services, limited resources and equipment, altered treatment protocols, and longer patient contact times. Medical practitioners on these expeditions must have increased clinical acumen, public health knowledge, and a cross-cultural understanding of their team members and the region's indigenous populations. They also must be able to provide and use diagnostic and management advice via telecommunications, devise and implement innovative practice methods, work beyond their normal scope of practice, make independent decisions, and assume increased responsibility. ${ }^{2,3}$

Remote expeditions are no longer just the purview of the military, mountain climbers, scientists, and explorers. 
Large corporations, especially those within the oil and gas industry, are sending an increasing number of teams into distant locales. As they push into ever more remote areas, these large teams often lack the ability to provide rapid evacuation to the definitive medical care that has become the industry standard. Corporate emphasis on employee safety and well-being (especially since the 1988 Piper Alpha gas and oil production platform disaster) normally includes having on-site nurses or medics and detailed plans (generally involving the use of helicopters) for the evacuation of patients needing further medical or surgical evaluation or treatment. When such rapid and assured medical evacuation is impossible, alternative medical support methods become necessary.

Providing medical care for remote expeditions requires extensive planning based on sufficient knowledge of emergency medical care and the operational environment, which can include ship or fleet operations, local medical facilities, geography and weather, the population to be treated, and local security. If done well, these plans should overcome most foreseeable difficulties, including those relating to the environment, medical fitness of personnel, medical command and control of the operation, communications, onboard medical equipment and medications, and appropriate professional training for the clinical staff.

Planners "need to cover all contingencies; from mild illnesses and disease...through to unforeseen events such as evacuating a seriously ill, injured or dead person." Although extensive planning goes into many of these enterprises, they often lack input from experts in emergency and remote medicine, leading to errors of omission and commission that may prove lethal. ${ }^{4}$

This paper sets forth 10 guidelines to help planners focus on the key, medically relevant elements of a workable remote healthcare system. Each guideline is followed by case examples from the author's Arctic and Antarctic experiences to illustrate outcomes when the planning process succeeded or failed, including how clinicians improvised solutions beyond the original plans' scope. Both of the author's extended remote expeditions used medical plans that, on occasion, clashed with the reality of conditions on the ground. The lessons drawn from these experiences may assist those planning medical support for future extended remote medical undertakings in austere environments.

\section{Optimize Workers' Fitness}

Every remote expedition should strive to obtain the healthiest possible workforce, and this should be emphasized in the medical plan. Reducing the incidence of illness "is largely a matter of determining medical standards for work [in remote environments] and ensuring that the criteria specified are met." remote expeditions do this within the constraints presented by cost, time, and the inherent limitations of physical examinations and medical tests. Screens for those at sea, for example, often follow one of several similar general screening formats that, aside from general wellness, evaluate limitations to perform specific functions. ${ }^{6,7}$ Large land-based operations generally follow their own, often detailed standards. However, because none of these screens has been shown to have any predictive validity, their primary purpose seems to be to screen for significant acute and chronic disease and for illegal drug use.

Screening medical examinations try to identify specific ailments or abnormalities that fall outside an expedition's standards. Yet it is often unclear what constitutes appropriate medical standards. As the workforce ages, more expedition team members may have significant preexisting health problems. Planners may need to anticipate providing urgent treatment for such common chronic illnesses as allergies, asthma, diabetes, epilepsy, cardiovascular disease and hypertension, and autoimmune diseases. ${ }^{1}$ It remains unclear how to best screen the many potential workers with prior injuries. ${ }^{2}$

Planners should recognize that the weakest part of medical screening is usually the physician-performed dental examination. Unless gross dental pathology exists, physicians are unlikely to identify potential problems. ${ }^{8}$ As a result, many individuals cleared for remote offshore work have dental caries, periodontal disease, or marginal restorations that may require intervention or evacuation. ${ }^{9,10}$ Indeed, over many decades, between about $5 \%$ and $15 \%$ of all offshore oil worker evacuations have been because of dental problems. ${ }^{8}$ Although many sources suggest careful predeployment dental examinations, a study comparing expeditions with and without such examinations failed to show a significant difference in the incidence of dental emergencies. ${ }^{8-10}$

Since one obvious benefit of medical and dental screenings is to identify, within providers' capabilities, acute and chronic medical conditions, it is vital that personnel not evade the requirement, as is described in the first case. Program physicians should, however, have the discretion to grant "waivers" in the many instances in which the nature of a medical or dental problem will neither compromise a person's job nor put coworkers or the project at risk.

Another vital component of planning for a healthy workforce is to provide personnel with a medical briefing several weeks or months before deployment. Topics 
covered should include environmental issues they may encounter, required vaccinations and prophylactic medications (eg, antimalarial agents), the need for an adequate supply of personal medications, and the project's emphasis on personal hygiene to prevent common gastroenteritis. $^{11}$

\section{CASES}

\section{Case 1}

Although the Antarctic program's plan had rigid medical criteria for both long-term employees and short-term official visitors, "VIPs" could bypass these requirements. One such visitor with a known serious cardiac arrhythmia exhibited ventricular tachycardia soon after arrival, requiring intensive treatment and evacuation. Another VIP visitor, who insisted on going to the South Pole Station (a physiological elevation that varies between 9,000 and 12,500 feet, depending on the ambient air pressure), ${ }^{12}$ needed immediate oxygen therapy and a wheelchair until he could be evacuated back to sea level. A third individual failed the initial screen, but was permitted to come to the sea-level station because his presence was considered vital to fixing the station's generator and he would only stay a short time; he had no medical problems during his stay.

\section{Case 2}

Although the Arctic project's plan required a "fitness-towork" examination and certificate, ${ }^{7,13}$ only about $10 \%$ of the deployed personnel had one. The planners decided that each person should have an antiquated Master 2-Step-Test, ${ }^{14}$ without either electrocardiogram (ECG) or blood pressure monitoring. The results, considered prognostically worthless, ${ }^{15}$ did not affect crewmembers' participation on the team. Nor did having a certificate preclude problems. One crewmember who had repeatedly obtained such a certificate "forgot" to mention his history of recurrent cardiac arrhythmias and ablations until he presented during the project with chest pain.

\section{Anticipate Treatable Problems}

Perhaps the most difficult question for remote expedition medical planners is to decide for which medical problems they need to prepare. Experts agree that "[i]t will never be possible or practical to deal with every possible situation, even with the best of equipment, so planning should look to managing the common things well and the more difficult medical crises in the best possible way. Improvisation is the name of the game." A good general rule is to evaluate medication and medical equipment supplies based on their cost versus the costs of lost work time, evacuation, personnel replacement, and expedition members' confidence that they will receive optimal medical care.

Any plan must address the most commonly encountered problems, nearly all of which will be medically "minor." One comprehensive survey of expedition illnesses and injuries, which covered 42,482 participantdays in multiple environments, reported 1564 incidents. Of these, 94\% were "minor" (uninterrupted participation, ie, return to work), $5 \%$ were "moderate" (1 day or more away or required local hospital assessment, ie, lost time), and $1 \%$ were "major" ("life-threatening condition, the need for immediate evacuation, or an incident resulting in death"). ${ }^{16}$

The most common medical conditions encountered on expeditions are gastrointestinal diseases, skin conditions, and minor trauma. Ophthalmological, dental, and respiratory problems provide the balance of cases, although motor vehicle crash injuries may be a significant factor, depending on the type of expedition. However, the probability of identified illness and injury varies with the particular environment and the expedition's reporting accuracy. ${ }^{2,4,5,8,16-21}$ Historical data compiled from multiple nations' Antarctic expeditions demonstrated incidence rates from 188 to more than 6600 physician encounters $/ 1000$ people per year. Injuries comprised $16 \%$ to $40 \%$ of these clinical encounters, with about $30 \%$ of injuries resulting from recreational rather than work activities. ${ }^{22}$ Similarly, on a US naval ship, recreational injuries accounted for 19\% of all injuries, with $25 \%$ requiring time off work. ${ }^{23}$ Older age seems to increase the incidence of serious illness but decreases serious injuries. ${ }^{5}$

Planners should normally base their medication and equipment stock on the most common presentations to emergency departments (EDs), as well as selected lesscommon, but high-risk, presentations that are survivable with remote treatment. An excellent resource for information about the more common illnesses and injuries is the ED section of the annual National Hospital Ambulatory Medical Care Survey, which, based on US hospitals, reports the incidence of presenting complaints by age and sex; diagnoses; procedures performed; blood tests, imaging, and other tests done; medications prescribed; and injury sites and causes. ${ }^{17}$ Planners can also incorporate recommendations from appropriate international bodies ${ }^{24}$ and environment-specific wilderness expedition medical reports. ${ }^{2,4,16}$ Unfortunately, occupational reporting, which would seem the most obvious data source, is too unreliable to use for this purpose. ${ }^{25}$

A component of medical planning is recognizing the significant costs associated with illness and injury on remote expeditions. These include costs for: 
- The incident: on-site first aid; use of and replacing supplies (often impossible to replace); evacuation

- Investigation/education: company and government accident investigation and paperwork (injuries); follow-up company-wide education (injuries and serious or recurrent illnesses)

- Replacement: hiring and transporting replacement worker; trainer/trainee time for new worker orientation

- Lost productivity: ill/injured worker's reduced productivity after treatment; immediate lost productivity of worker and those involved in treatment/stabilization/evacuation; work disruptions. ${ }^{26}$

To simplify the process, a number of computer programs automatically calculate the cost to expedition planners of various illnesses and injuries. A free cost calculator, available through the US Department of Labor, estimates, for example, that the total direct and indirect costs for a myocardial infarction is $\$ 233,490$; for a concussion, $\$ 166,708$; for multiple trauma, $\$ 161,550$; and for fractures, $\$ 93,363 .{ }^{27}$ These costs, of course, are for the normal, rather than the remote, work environment.

\section{CASE}

In the Arctic, although the initial planners may have anticipated some treatable medical problems, the final plan ignored them. In large measure this was because, contrary to basic practice, the expedition's remote medical plan was devised by one group in isolation, the basic medical supplies and equipment were purchased by a second group based on their own (Norwegian ship) standards, and a third group provided additional medications and equipment (primarily to address advanced cardiac life support). The third group also arranged for external consultations and the medical evacuation of patients once they were on shore. Despite the extremely remote location and the severe environment, none of these groups coordinated their plans with the others. Instead, they based their individual plans on a misunderstanding of the overall expedition plan, the environmental conditions, and the local work setting.

A few of the resulting discordances were:

- Caring for musculoskeletal injuries, including having "splinting materials," was initially required. No materials were available and had to be obtained through the good graces of the hospital in the ultimate departure port.

- The ability to perform wound repair was anticipated, but no needle holder was supplied.

- At least 24-hour ventilatory support was specified, but no long-term (and sparse short-term) paralytics were provided.

\section{Stock Appropriate Medications}

Planners should select medications based primarily on the expedition's anticipated illnesses and injuries. Calculating the expedition's supply of each medication depends on:

(the probable number of patients needing the medication) $\times$ (the number of doses needed to treat one patient).

Additional factors to consider include medication costs, fewest side effects, fewer doses needed to achieve effect, available storage space (including refrigeration and locked/controlled drug storage), shelf-life, therapeutic index (safety), expedition clinician's experience, and the expedition members' cultural norms (particularly for over-the-counter [OTC] medications). Conflicting and changing legal requirements may hamper providing an appropriate expedition formulary, such as when the expedition ship must follow its flag-state medication stocking regulations.

Whenever possible, planners should stock first-line medications for the commonly anticipated ailments, with the understanding that the standards designating what the first-line medication is may vary. A rule of thumb is to supply medications with multiple possible uses whenever possible, such as diphenhydramine and epinephrine.

Repeatedly, planners fail to supply sufficient medications for common problems that patients would normally treat with OTC medications. Upper respiratory infections, for example, often spread quickly through an expedition's closed environment, exhausting the generally meager (and not easily replaceable) stock of most expeditions. In some cases, the infections and their resultant productivity loss could be decreased by having the medical staff delay symptomatic (even if afebrile) expedition members from joining the expedition until they recover.

In specific environments, medical planners will need to provide additional medications. For example, spare antimalarial agents will be needed where malaria is endemic, and seasickness medication, lanolin hand cream, and sunscreen are useful at sea. Snake antiserum and rabies vaccine may be appropriate in high-risk areas, and diving expeditions should stock otitis externa medications and oxygen. High altitude ventures may need oxygen, acetazolamide, dexamethasone, nifedipine, and salmeterol. ${ }^{1}$

To improve safety and dispensing ease, planners should assure that suppliers only provide medications labeled with the generic name, dose, and indications for use in English or the provider's language. If necessary, the container should have an extra label with this information. 


\section{CASES}

\section{Case 1}

The Arctic support vessels each carried a variety of medications approved by their maritime authority. Their supplies were usually minimal, and several had medications that many countries had banned as being too dangerous to use, such as the analgesic/antipyretic metamizole, also known as dipyrone. Although the expedition's planners expected providers on the research vessel to treat the support vessels' crewmembers, this proved to be difficult. Such care often required improvising treatment when the appropriate medication either was not stocked or could not be transferred to the patient's vessel. (Transfers between ships are dependent on weather conditions and distance.) Planners have agreed to provide each support vessel on future expeditions with a standardized medical kit so that they will have appropriate medications when instructed to use them.

\section{Case 2}

When a 31-year-old Arctic crewman presented with herpes zoster over his right shoulder (C-4 dermatome), providers discovered that no oral acyclovir was available. Needing to improvise, they researched alternatives and found a 100-patient comparison study that demonstrated the successful use of topical acyclovir, which they then administered. ${ }^{28}$ In this case, however, given the patient's young age, minimal symptoms, and truncal involvement, another option was not to treat because the risk of complications was low.

\section{Provide Appropriate Equipment}

Planners should use available information, including prior expeditions' experiences, to determine the probability of needing medical equipment in particular environments. ${ }^{11,21,24}$ The major criterion is to provide what clinicians will need to diagnose and treat common problems, to convert patient evacuations to restricted duty, or to convert emergency evacuations to scheduled departures. This includes most equipment required for ophthalmologic, otolaryngologic, dental, traumatic, orthopedic, and extraction/evacuation situations.

The provision of additional equipment for advanced airways, ventilation, laboratory testing, and diagnostic imaging will depend on the expedition's size, the remoteness of the location, the practitioner's skills, and the planners' aversion to risk and uncertainty. Planners may be more willing to supply future remote expeditions with ultrasound and x-ray machines as their size and cost decrease. $^{29,30}$ The limiting factor to using ultrasound, however, is that it requires either a clinician with the ability to independently perform the examinations and interpret the results or sufficient bandwidth to transmit real-time images to experts. Imaging equipment also should include a digital camera to send e-mail images to consultants.

In all cases, planners should opt for the simplest equipment that performs well. Fortuitously, such equipment is often the least expensive. For example, instead of purchasing multiple pieces of complex laboratory equipment, planners can supply the simple handheld bedside test equipment that most emergency and intensive care clinicians routinely use. They should function well in the same temperature-controlled settings that other laboratory equipment requires. The following are guidelines for selecting simple, effective equipment:

- Easy to use

- Portable for use at multiple local and remote sites

- Familiar to clinical providers

- Rugged (field-tested in EDs, intensive care units, and prehospital settings)

- Fewest possible reagents used, especially refrigerated reagents

- Clear instructions in language that providers can understand

- Appropriate for the setting, eg, equipment with available bandwidth, adequate refrigeration for reagents, minimal vibration for sensitive laboratory tests, or reliable electricity, as needed to run an oxygen concentrator. Remember that battery-operated equipment, such as a monitor-defibrillator, must eventually be recharged - Adequate for the intended purpose, eg, an x-ray machine that will take both a chest and an abdominal radiograph, or an ultrasound with probes capable of diagnosing intraabdominal pathology, pneumothoraces, and venous thrombosis

All medical plans for remote expeditions must include time to assemble, install, train on, and test all equipment before departing. This avoids having the clinicians discover, when already in the field, that equipment does not work, vital equipment parts are missing, or they cannot operate unfamiliar equipment (as the manual is missing or not in the provider's language).

\section{CASES}

\section{Case 1}

Arctic planners supplied almost no dental equipment and supplies, although researchers have long recognized that dental problems constitute common reasons for evacuations from remote expeditions and have advised providing at least dental first aid kits. ${ }^{10}$ When 2 crewmembers 
presented with 3 carious, fractured molars, providers had to improvise virtually everything they needed to perform extractions: a dental chair from an office chair covered in plastic bags, protective gowns from plastic bags, and a functioning suction container from cannibalized equipment. After compounding a lidocaine-epinephrine solution, ${ }^{31}$ providers used their personal headlamps to compensate for the surgical lamp that could not be positioned correctly, and borrowed "dental" tools from the ship's electrician, instrument room, and engineers. All 3 teeth were successfully extracted and the patients healed well.

\section{Case 2}

When a 49-year-old expedition crewmember presented with a probable ureteral stone, health providers had to assess the need for his evacuation. After confirming microscopic hematuria and normal laboratory tests, they conducted a renal ultrasound, which showed normal kidneys. At that point, the ultrasound machine, designed to transmit the real-time image had there been sufficient bandwidth, stopped working-permanently. They then tried to take an abdominal radiograph, in case the stone was radiopaque, to assess the chance of it passing. Only then did they discover that the x-ray system the planners had supplied took neither chest nor abdominal radiographs; the system was apparently designed to obtain only extremity radiographs. Eventually, the providers found that they could transfer the ultrasound program onto a generic laptop computer and use the probe through the USB port, making the now-portable machine much more useful.

\section{Case 3}

Anticipating the possibility that multiple aeromedical missions might occur simultaneously or that equipment might be unavailable owing to malfunction or maintenance, Antarctic planners supplied sufficient redundant equipment to compensate.

\section{Provide Adequate Logistical Support}

Nearly all extended remote expeditions must be periodically resupplied, although the supply chain may be tenuous and sporadic. Given the situational constraints and the need for frontline medical providers to have the tools they need, planners should ensure that expedition logistics function as smoothly as possible using proven healthcare supply systems. ${ }^{32}$ Some logistical considerations for a remote expedition team's medical support include:

- A clear and responsive logistical chain

- A system that providers can easily access to order supplies and equipment ("pull system")
- Prompt delivery of requested items, including the necessary components to make them functional

- Timely responses to requests, including expedited responses for critical medications and equipment

- Cognizance of demand variability, accessibility of the expedition, and delays for customs clearance (note that transporting medical materials, especially controlled substances, through or into some countries may be a difficult and time-consuming process)

- Maintaining the cold/warm chain for medications and reagents during transport

\section{CASES}

\section{Case 1}

Although providers had made requests at the start of the project, 2 months into the Arctic expedition, most requested medications and equipment still had not arrived, and some on-site equipment did not function. Unusable and missing equipment included personal protective equipment, dental equipment, oxygen connections to power a ventilator, and ECG electrodes. Both cold- and warm-chain medications that needed to be kept cold (or not frozen) arrived without temperature controls and had to be discarded. Ultimately, the expedition obtained some medical supplies through a local Greenland source and from a medic who purchased some medications while on leave.

\section{Case 2}

In Antarctica, the logistical system functioned well when an unexpected influenza pandemic occurred. Medical staff requested vaccine for the entire expedition that, although it was in short supply, was rapidly provided, along with antiviral medications.

\section{Provide Adequate Medical Communications}

Planners must anticipate the need for reliable medical communications between on-site providers and off-site consultants, administrative support sites, and various expedition sites, such as field camps, ships, and distant fixed facilities. Although remote expeditions have increasingly sophisticated communication options, none of them are $100 \%$ reliable. ${ }^{1}$ The likelihood of equipment failure is much greater if the equipment is not tested before departure.

At a minimum, medical plans for large extended remote expeditions should include having Internet access with sufficient bandwidth to transmit digital images of patients' affected body parts or radiographs using e-mail. In areas with cell phone coverage, these devices can often be used to send clinical images. If it is necessary to 
send real-time ultrasound images to a consultant (as when the on-site provider lacks sufficient ultrasound experience), significantly more bandwidth will be required. If providers will need to obtain real-time consultation or patient-management assistance, they should plan to use a mobile video camera and Voice over Internet Protocol (VoIP) software. Again, this will require a reliable Internet connection with a significant amount of bandwidth.

Planners must also compensate for geographical constraints or logistical factors that hamper communications, such as the limited communications satellite availability at the poles and in mountainous areas, caves, and mines. With fewer geographical constraints and available repeaters, some groups may use amateur (ham) radio, although maintaining confidentiality while using these widely available radio bands may be problematic.

Medical plans for remote expeditions usually focus on communication technology while overlooking optimal information transfer. As NASA discovered early in the space program, when dealing with remote teams that may encounter a wide variety of unforeseen problems, it is optimal to have a very limited cadre of knowledgeable professionals serving as their contacts. ${ }^{33}$ That way, any discussions, disagreements, and confusion among administrators at the home base can be resolved before giving a designated communicator transmit information. Omitting this element results in multiple people, with different areas of responsibility and levels of authority, all providing the expedition medical staff with partial or conflicting information. This diminishes the field team's confidence in the system, causes confusion, and generates hostility. The British Antarctic Survey, having learned this, includes medical communication instruction in their orientation because they believe that the "system depends upon the existence of clear and precise communications." 34

\section{CASES}

\section{Case 1}

In the Arctic, planners purchased expensive ultrasound equipment and a special patient examination camera for real-time examinations with the expectation that a distant radiologist or specialist consultant could guide and interpret examinations. Once on site, however, providers discovered that there was not enough bandwidth to allow transmission from either device. The clinicians also could not use their improvised alternative because the expedition's Internet service provider (ISP) would not allow the use of VoIP. As a last resort, the clinicians began relying on photographs sent via e-mail for consultations; however, had the clinicians needed assistance with ultrasound, this solution would not have worked. Hopefully, in the future, the planners will check for such potential problems before deployment.

\section{Case 2}

The Antarctic plan included the use of real-time telemedical supervision from the main base at McMurdo Station to the South Pole Station. When an individual at the South Pole suffered a cardiac event, senior emergency physicians observed the resuscitation and provided real-time direction from the base station. The patient lived. Fortunately, this occurred when satellite communication was available to the South Pole, which has tenuous coverage owing to its geographic position. However, the medical plan included alternative telephone communication that, although less optimal for this purpose, was always available.

\section{Case 3}

Although the Arctic medical planners expected physicians on the research vessel to provide medical assistance to the support vessel crews, they provided no communication protocol for this. The result was confusion, delays, and a loss of patient confidentiality. After support vessel captains began sending messages requesting physician assistance without including relevant information, the clinicians demanded that in nonemergency situations they provide a description of the situation, vital signs, physical findings, and e-mailed photographs. That way, the physicians could determine whether they could provide telephonic advice or they would need to go see the patient or have him transferred to their ship. Unfortunately, even this system failed when 2 support ships carrying 3 patients who needed followup lost their e-mail service because they failed to pay their ISP bill. The (inadequate) solution was to communicate via open-channel very-high-frequency radio and bridge phones, broadcasting confidential patient information to many unintended sites.

\section{Know the Environmental Limitations on Patient Access and Evacuation}

In potentially hostile environments, the assumption that access to patients, such as those on other ships or at distant field camps or at distant fixed sites, will always be easy is a mistake. Going blindly into a remote, hostile environment portends trouble. When planning medical support for an expedition, consider environmental factors and local geography as these may determine the difficulty of accessing a patient for treatment or evacuation. ${ }^{1,11}$

Planners should evaluate patient accessibility in terms of weather, local environmental conditions, and the 
availability of transportation. Most remote locations have periods of extreme weather, such as high winds, severe storms (rain, snow, or sand), and life-endangering temperatures, which may delay access to a patient for evaluation and treatment. Similarly, high or ice-laden seas, wildfires, volcanic activity, or unsuitable geography (too rough or too far away) can limit the provision of patient care. In addition, planned transportation may be unavailable because of mechanical problems, environmental conditions (such as a helicopter's inability to fly in high winds or small boats to operate in open seas after dark), or a lack of trained personnel.

Plans should always include provisions for the emergency evacuation of patients, even when there are highlevel, on-site facilities and healthcare providers. Remember that the same factors that may limit access to a patient can also prevent evacuations. Ho et $\mathrm{al}^{21}{ }^{21}$ describing the Juneau Icefield expeditions, wrote, "All such evacuations are therefore subject to myriad unpredictable variables, including weather, accessibility to adequate landing zones, radio communications, and helicopter availability. Accordingly, medical staff must be prepared to provide temporary treatment for up to several days [or longer] until rescue can be achieved." Always plan to provide sufficient medications and equipment to care for patients when evacuations are delayed.

Leaders should inform potential team members of the increased risk of joining the expedition. It is important that participants have realistic expectations concerning treatment and evacuation, including recognizing that death could occur from conditions that might otherwise have been survivable in optimal medical settings. ${ }^{1}$ A hallmark of remote expeditions is that significant time and distance always separate them from centers with adequate surgical or intensive care facilities. ${ }^{1,34}$

To facilitate evacuation decisions, planners must provide clinicians with an accurate assessment of local healthcare facilities and the quality of available medical care. ${ }^{11}$ As part of training, providers must be familiar with any equipment they may need to use and any aspects of evacuation procedures in which they will be involved.

The medical plan must also address who should escort a patient, if that becomes necessary. A similar consideration is what to do if a healthcare provider needs medical evacuation. Who will provide medical care for other expedition members during their absence? Planners should consider whether a second clinician should accompany the expedition. If so, that individual must have sufficient skills to care for team members solo. ${ }^{11}$

A goal for remote expedition medical planners is to reduce the need for emergency medical evacuations. Ideally, an on-site physician with adequate equipment (especially imaging equipment), medications, and support-and, ultimately, an unaccompanied nurse or medic with the same materials and telemedical support-could stabilize most patients enough (or give a clear enough diagnosis) to make an emergency evacuation unnecessary.

Of course, planners, expedition leaders, transport personnel, and receiving facilities should test the evacuation system and equipment before departure. This should involve at least a tabletop exercise with tests of the various communication and decision-making elements.

\section{CASES}

\section{Case 1}

The Arctic planners did not foresee the need to transfer either medical personnel to the support vessels or patients to the research vessels, which offered imaging, ECG, and laboratory equipment, for evaluation and treatment. In not doing so, they also failed to consider how such transfers might safely be accomplished. Strict industry-wide operational and safety standards restrict the use of small boats and the "frog" when seas are rough or visibility is limited. However, several times during the project, just such transfers were needed, necessitating the use of small boats over frigid Arctic seas or, as the initial planners had erroneously suggested, a frog personnel transfer system (a personnel-carrying basket passed between boats using a crane). In fact, this fleet had never used or carried a frog. Additionally, although nearly constant daylight existed at the beginning of the project, it decreased to less than 12 hours a day for the last month. On several occasions, unsafe sea conditions prohibited small boat operations for up to a week at a time, meaning that medical personnel, patients, medications, and equipment could not move between vessels. During that time, care was limited to providing advice via e-mail, phone, or radio.

\section{Case 2}

Antarctic planners carefully considered medical alternatives when devising a novel transpolar land expedition. The caravan necessarily traversed an area where no medical access was possible because of the distance from any helicopter refueling station and the unsuitability of the terrain for a fixed-wing plane to land. Their plan was to provide telephonic medical support to designated companions, one of whom was a paramedic, while they transported the patient to an accessible evacuation area. Fortunately, no medical care was needed during the trip. 


\section{Use Qualified Providers}

Although good planning helps prepare for and avoid many foreseeable medical and logistical problems, excellent clinicians are needed not only to provide care according to the plan but also to deal with unforeseeable events. These practitioners must possess the appropriate clinical experience (including public health), be able to make independent decisions, and be willing to improvise solutions to novel problems. Because most expeditions include diverse personnel and may involve work with local populations, the ability to optimize cross-cultural interactions is helpful.

Ideally, expedition doctors "should have previous experience in emergency and prehospital care, expedition medicine, and the ability to operate effectively in remote environments. There is not yet a minimum qualification for expedition medics." 16 Providers must be able to confidently diagnose and treat a diverse range of medical complaints and conditions, from minor ailments to multiple patients with major trauma. ${ }^{2}$ They also must be familiar with conditions related to the expedition's environment, such as altitude-related illness, temperaturedependent injuries, barotrauma, or tropical diseases. ${ }^{1}$ On all but the largest expeditions, they normally must also monitor food and water safety for the team.

Clinicians also must have training on the expedition's imaging, laboratory, and telemedicine equipment because each equipment brand or model has individual peculiarities that should not be first encountered in the field. They should also have training in dental care, food and water safety monitoring, and any unique evacuation equipment or procedures that the expedition might use. A model for this, which may be excessive for emergency medicinetrained physicians, is the course provided to British Antarctic Service medical officers. They receive instruction in "first-aid, diving medicine, radiography and radiology, medical communication, anesthetics and analgesics for remote places, plastering, dental first-aid, environmental health and introductory research methods." 34

Finally, providers must describe their credentials and the expedition's medical capabilities during the predeparture orientation for team members. Primarily, they must explain that "expedition members cannot expect to receive the same 'standards of care' as they may expect in a developed country, but they will need to expect to receive a similar standard to that of any competent medical officer in a similar situation."1

\section{CASES}

Case 1

Remote expedition planners would never consider idiopathic persistent singultus (hiccups) on a list of possible patient presentations. Yet, when a 40-year-old Antarctic expedition member presented with a recurrence of this problem, which had plagued him several times during the past decade (with negative medical evaluations), that had persisted for 48 hours, the providers had a choice of evacuating him or improvising a remedy that would allow him to continue working. They successfully employed a little-used treatment, intravenous lidocaine. The singultus immediately resolved and did not recur over the following 3 months. ${ }^{35}$

\section{Case 2}

Providers in the Arctic were not told that planners expected them to have completed training in the accepted systematic method to monitor food safety and preparation areas (Hazard Analysis and Critical Control Points [HACCP]). However, using knowledge gained from prior expeditions, the providers identified and corrected deficiencies in food safety monitoring, which included improving methods to keep food warm while in the self-serve trays and monitoring food temperature during the preparation and serving process.

\section{Arrange for Knowledgeable and Timely Consultations}

Medical plans for remote expeditions must anticipate that even the most experienced healthcare provider will occasionally need consultants' help, even if they have online medical references available. Specialists can assist providers with patient diagnosis or management and help them to decide whether patients require evacuation.

Some expeditions use consultation groups that employ physicians-in-training (residents) to field questions. This system may suffice for most consultations requested by paramedical personnel, but it is woefully insufficient when experienced physicians require consultations. A better system, used by some offshore facilities, is to have a medical coordinating unit with emergency medicine-experienced base doctors familiar with remote medical problems and the offshore provider's capabilities, and who are experienced in providing long-distance clinical advice. ${ }^{8,18,34}$

No matter what type of consultation system is used, planners should assure that the appropriate senior specialists will be immediately available. These should include emergency physicians, dentists/oral surgeons, orthopedic surgeons, radiologists (if ultrasound or x-ray will be available), dermatologists, ophthalmologists, otolaryngologists, general internists, intensivists, infectious disease specialists, gynecologists (if women will be on the expedition), neurosurgeons, cardiologists, 
psychiatrists, general surgeons, and pediatricians (if children from the local population will be treated).

\section{CASES}

\section{Case 1}

The Arctic consultation plan relied on talking with the physician at the closest Greenland town. It was assumed that this individual would provide advice or be a link with specialty consultants elsewhere in Greenland or Denmark. On testing this system, providers discovered that the consultant could neither speak English nor hear very well over the phone. His nonmedically trained wife was expected to act as an intermediary. The plan then shifted to the main Greenland hospital from which specialty consulting advice, when received, took 2 weeks or more. At that point, the clinicians began requesting consultations, via e-mail accompanied by relevant photographs, from senior specialist colleagues from The University of Arizona College of Medicine in Tucson, Arizona. The consultants invariably responded within 12 hours. We subsequently got excellent consultation support from the local Greenland dentist.

\section{Case 2}

In Antarctica, the consultation plan instructed providers to contact a major teaching hospital's emergency department, where a senior resident or attending emergency physician would respond. Tests of the system demonstrated that obtaining specialty consultation would be a slow and laborious process - which would usually lead to a consultation by a specialty resident rather than by an attending physician. Because the clinical staff requesting these consultations usually had more experience than these consultants did, the expedition's clinicians ended up relying on specialty colleagues at their home university for consults.

\section{Establish and Distribute Rational Administrative Rules}

Every remote medical expedition has administrative rules or guidelines; the larger the sponsoring organization, the more rules that exist. Some of these rules stem from government guidelines, such as the Occupational Safety and Health Administration's reporting requirements for on-the-job injuries. Others are specific to that organization. Before an expedition starts, planners must be certain that all medical providers and the team leaders know the rules applicable to the medical unit and that these rules cover all appropriate areas, including access to confidential patient information. ${ }^{11}$

Complex operations often have multiple and conflicting lines of authority that may unnecessarily complicate the operation and strain even the most elegant plan. On large remote expeditions, the on-site manager, the client representative, the general contractor for services, the medical contractor if a different entity, and the client that ultimately pays the bills may each believe that they have ultimate authority over medical matters. The goal of providing optimal patient care may be lost when so many people, with contradictory and conflicting interests, spending and profit considerations, and different ideas and knowledge become involved in every decision about an expedition's medical activities. It is the planners' responsibility to clarify the lines of authority before the expedition deploys; this ensures that decisions are made using a clear and coordinated process rather than by multiple parties trying to cope with a confused and complicated situation. One solution is to make a single medical administrator responsible for coordinating a remote expedition's healthcare. That individual should have final decision-making authority regarding personnel, equipment, medications, and reporting requirements.

Planners must also consider who will make the decision to evacuate a patient, and how such decisions are made. There must be a clear algorithm for providers to follow; the process should not be devised ad hoc when the situation arises and the team is under pressure to "do something." Although these decisions must ultimately rest with those who control the evacuation resources, which may include people, animals, ships, helicopters, and planes, they must be based on the clinicians' evaluation of their patient, the evacuation risk estimate, and the knowledge of the appropriate and available regional medical facilities.

Planners should also decide whether and to what extent expedition medical staff will treat the local population. If so, the plan should make available appropriate medications and equipment, including those for children and pregnant women. As a matter of course, "life, or limb, saving treatment should always be performed" if the providers are able to do so. ${ }^{11}$

\section{CASES}

\section{Case 1}

Providers identified an apparent Norovirus outbreak on an Arctic support vessel and responded appropriately using a plan that they had developed before the expedition. Because planners had not clearly delineated the lines of authority and responsibility, the client's offsite medical team inappropriately intervened after the problem was resolved. Continuing a pattern of uncoordinated actions among the expedition partners, the client's medical team tried to initiate actions that were redundant (ie, taking actions that had already occurred), untenable (ie, testing biological samples on board the 
ship), and illegal (ie, transporting biological samples internationally for testing without proper authorization).

\section{Case 2}

Although rules strictly forbid anyone from entering the South Pole station unless they are program participants, there is an explicit exception for life- or limb-threatening medical emergencies. When a visiting tourist suddenly collapsed with a cardiac emergency while standing next to the ceremonial South Pole, he was immediately brought into the station's medical facility, successfully resuscitated, and monitored for several days before being evacuated to a tertiary-care facility in New Zealand.

\section{Conclusions}

Remote extended expeditions often support scientific research and commercial resource exploration or extraction being conducted in hostile environments. Medical support for such expeditions is inherently complex and requires indepth planning. To be successful, the planning process must include substantial input from clinicians with experience in remote, emergency, and prehospital medicine and from personnel familiar with the proposed working environment. Use of the guidelines discussed in this paper will help ensure that planners consider all necessary, medically relevant elements before launching an extended remote expedition.

The 10 key elements of a workable remote healthcare system are to:

1. Optimize workers' fitness

2. Anticipate treatable problems

3. Stock appropriate medications

4. Provide appropriate equipment

5. Provide adequate logistical support

6. Provide adequate medical communications

7. Know the environmental limitations on patient access and evacuation

8. Use qualified providers

9. Arrange for knowledgeable and timely consultations

10. Establish and distribute rational administrative rules

Planners using these guidelines may better be able to generate a strategy that optimizes the participants' health benefits, the expedition's productivity, and the expedition sponsor's cost savings.

\section{References}

1. Shaw MTM, Dallimore J. The medical preparation of expeditions: the role of the medical officer. Travel Med Infect Dis. 2005;3:213-223.
2. McIntosh SE, Leemon D, Visitacion J, Schimelpfenig T, Fosnocht D. Medical incidents and evacuations on wilderness expeditions. Wilderness Environ Med. 2007;18:298304.

3. Smith JD, Margolis SA, Ayton J, et al. Defining remote medical practice: a consensus viewpoint of medical practitioners working and teaching in remote practice. Med J Aust. 2008;188:159-161.

4. Anderson SR, Johnson CJH. Expedition health and safety: a risk assessment. J R Soc Med. 2000;93:557-562.

5. Norman JN, Ballantine BN, Brebner JA, et al. Medical evacuations from offshore structures. $\mathrm{Br} J$ Ind Med. 1988;45:619-623.

6. International Labour Organization, International Maritime Organization. Sectoral Activities Programme; ILO/IMO/ JMS/2011. Proposed revised Guidelines on the Medical Examinations Of Seafarers. Report for discussion at the joint ILO/IMO meeting on medical fitness examinations of seafarers and ships' medicine chests (26-30 September 2011). Geneva, 2011. Available at: http://www.ilo.org/ wcmsp5/groups/public/@ed_dialogue/@sector/documents/ meetingdocument/wcms_162824.pdf. Accessed June 15, 2013.

7. British Department for Transport, Maritime and Coastguard Agency. Approved Doctor's Manual: Seafarer Medical Examinations, January 2010. United Kingdom: MCA, 2010. Available at: http://www.dft.gov.uk/mca/ mca_doctors_manual_links_.pdf. Accessed December 22, 2012.

8. Norman JN. A comparison of the patterns of illness and injury occurring on offshore structures in the northern North Sea and the stations of the British Antarctic Survey. Arctic Med Res. 1991;Suppl:719-721.

9. Hahn MJ. The dental status of workers on offshore installations in the UK oil and gas industry. $\mathrm{Br}$ Dent $\mathrm{J}$. 1987; 163:262-264.

10. Duffy B. Dental problems in the offshore oil and gas industry: a review. Occup Med (Lond). 1996;46:79-83.

11. Nicol ED, Dhillon S. Expedition medicine. J R Army Med Corps. 2005;151:264-271.

12. United States Antarctic Program. Physiology of altitude. Available at: http://www.usap.gov/travelAndDeployment/ contentHandler.cfm?id=1819. Accessed March 26, 2013.

13. UK Offshore Operators Association Ltd. Health Advisory Committee Guidelines for Medical Aspects of Fitness for Offshore Work: A Guide for Examining Physicians. Issue 5. London, UK: UKOOA, July 2003:34.

14. Master AM, Oppenheimer ET. A simple exercise tolerance test for circulatory efficiency with standard tables for normal individuals. Am J Med Sci. 1929;177:223-243.

15. Wackers FJ. Customized exercise testing. J Am Coll Cardiol. 2009;54:546-548.

16. Lyon RM, Wiggins CM. Expedition medicine-the risk of illness and injury. Wilderness Environ Med. 2010;21: 318-324.

17. Ambulatory and Hospital Care Statistics Branch, US Bureau of the Census. National Hospital Ambulatory 
Medical Care Survey: 2009 Emergency Department Summary Tables. Available at: http://www.cdc.gov/nchs/data/ ahcd/nhamcs_emergency/2009_ed_web_tables.pdf. Accessed December 17, 2012.

18. Norman JN. Medical care and human biological research in the British Antarctic Survey Medical Unit. Arctic Med Res. 1989;48:103-116.

19. Tonna JE, Lewin MR, Hahn IH, Platts-Mills TF, Norell MA. A prospective, multi-year analysis of illness and injury during summer travel to arid environments. Wilderness Environ Med. 2009;20:107-112.

20. Richardson KE. Diving expedition medicine-the Coral Cay Conservation experience. Div Hyperbaric Med J. 2007;37:189-197.

21. Ho C, Adema G, Davis D, Stinson M. A seven-year experience in expedition medicine: the Juneau Icefield Research Program. J Emerg Med. 2003;25:257-264.

22. Cattermole TJ. The incidence of injury with the British Antarctic Survey, 1986-1995. Int J Circumpolar Health. 2001;60:72-81.

23. Krentz MJ, Li G, Baker SP. At work and play in a hazardous environment: injuries aboard a deployed U.S. Navy aircraft carrier. Aviat Space Environ Med. 1997;68:51-55.

24. World Medical Association. International Medical Guide for Ships. 3rd ed Geneva, Switzerland: WHO Press; 2007.

25. Azaroff LS, Levenstein C, Wegman DH. Occupational injury and illness surveillance: conceptual filters explain underreporting. Am J Public Health. 2002;92:1421-1429.

26. New Zealand Accident Compensation Corporation (ACC). Cost calculator for injuries and illness. Available at: http:// www.acc.co.nz/preventing-injuries/at-work/injury-cost-cal culator/index.htm. Accessed December 25, 2012.
27. US Department of Labor, Bureau of Labor Statistics. OSHA's \$afety Pays Program. Estimated Costs of Occupational Injuries and Illnesses and Estimated Impact on a Company's Profitability Worksheet. Available at: http:// www.osha.gov/dcsp/smallbusiness/safetypays/estimator. html. Accessed December 25, 2012.

28. Prasanna BR, Pinto J, Kamat N. Comparison of therapeutic efficacy of acyclovir 5\% cream with silver sulphadiazine $1 \%$ cream in herpes zoster. Indian J Dermatol Venereol Leprol. 1992;58:243-245.

29. Hope B. What's next for portable ultrasound? Available at: http://www.itnonline.com/article/what\%E2\%80\%99s-nextportable-ultrasound. Accessed December 25, 2012.

30. Istook M. Palm-sized Star Trek tech may be closer than you think. Yahoo! News. Available at: http://news.yahoo. com/blogs/future-is-now/palm-sized-star-trek-tech-may-clo ser-think-210316301.html. Accessed December 25, 2012.

31. Iserson KV. Improvised Medicine: Providing Care in Extreme Environments. New York, NY: McGraw-Hill Publishing; 2012:158, 178.

32. US Agency for International Development. The Logistics Handbook: A Practical Guide for the Supply Chain Management of Health Commodities. Arlington, VA: USAID; 2011.

33. National Aeronautics and Space Administration. CAPCOM: Capsule Communicator. Available at: http://space stationlive.jsc.nasa.gov/additional/index.html\#capcom. Accessed January 2, 2013.

34. Norman JN, Laws RM. Remote health care for Antarctica: the BAS medical unit. Polar Rec. 1988;24:317-320.

35. Iserson KV. Improvised Medicine: Providing Care in Extreme Environments. New York, NY: McGraw-Hill Publishing; 2012:475. 